\title{
The Influence of Emotions on Keystroke Patterns in Android Platform using Auditory Stimuli - An Experimental Study
}

\author{
Prajakta M. Dhamanskar ${ }^{1}$, Lillita Rhea D'souza ${ }^{2}$, Chaitrali Gandhi ${ }^{2}$, Naman Malik ${ }^{2}$ \\ ${ }^{1}$ Assistant Professor, ${ }^{2}$ Student \\ Fr. C.R.C.E. \\ Bandra, Mumbai
}

\begin{abstract}
Keystroke Dynamics or typing dynamics refers to the method of identifying or confirming the identity of a person based on the typing pattern by checking the various timing information obtained when a key is pressed and released. It has been hypothesized that a user's keystroke patterns change according to his/her emotions. However, there were only limited investigations about the phenomenon itself in previous studies. The work in this paper is based on the use of auditory stimuli to check the influence of keystroke patterns and its variations according to the emotions of an individual. The advantages of using this method are that the data collected through this approach is non-intrusive and easy to obtain. The proposed system is of a controlled experiment to collect keystroke data from multiple subjects in a variety of emotional states induced by International Affective Digitized Sounds (IADS) using an Android Application. To examine the data collected, Two-way Valence (3) x Arousal (3) ANOVAs is applied. The work in this paper aims to prove that keystroke duration and latency are influenced by valence and arousal.
\end{abstract}

\section{General Terms}

Keystroke Dynamics, Emotional Influence

\section{Keywords}

Android, Arousal, Auditory stimuli, Authentication, Emotions Recognition, Keystroke Dynamics, Valence

\section{INTRODUCTION}

Authentication is the act of confirming the truth of a person by use of a single piece of data claimed true by an entity. It is one of the primary ways of security in any device. Especially in today's age when every single application is digital and available to the masses instantly. Authentication is classified in three sections: -1 . Knowledge based - This method requires the knowledge of private information of an individual e.g. Password or PIN. 2. Object based-A Person can be authenticated using his/her personal object which is scanned by an image capturing device and the template is stored which is compared each time the user tries to access the system e.g. ATM card 3. Biometrics based- This is based on human unique characteristics to identify an individual. However, biometric characteristics can be further divided in two main classes:

Physiological: Face, Fingerprint, Hand, Iris.

Behavioral: Keystroke, Signature, and Voice.

Keystroke Dynamics is one of the newest and emerging field of authentication. Keystroke dynamics refers to the automated method of identifying or confirming the identity of an individual based on the manner and the rhythm of typing on a keyboard. The keystroke rhythms of a user generate a unique biometric template of the individual's typing pattern for authentication.

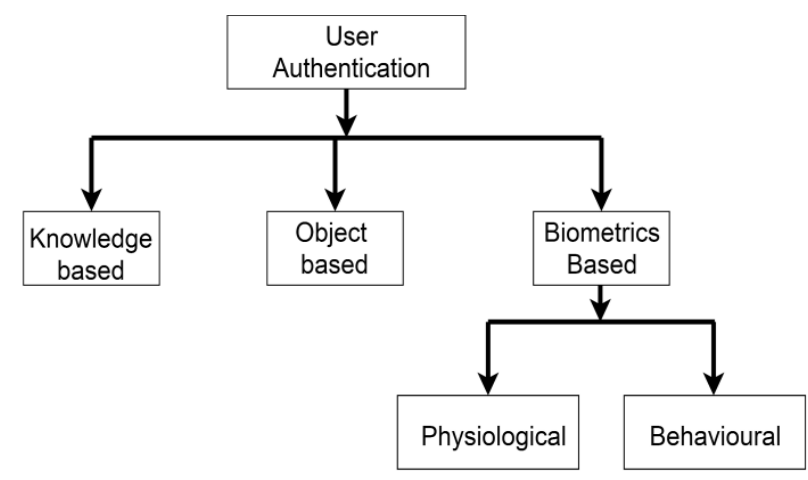

Fig 1: Classification of User Authentication

The key measurements used to determine the unique typing rhythm of a user are as follows: -

Keystroke Duration- The time period between key press and key release.

Keystroke Latency- The time period between key release and next key pressed.

The recorded data is then processed through a unique algorithm which determines the user pattern for future comparison. Although there are several studies on PC systems, there are a few related works performed on smart phones. Obviously, the typing patterns on touchscreens will not be similar to a normal keyboard. Due to this Android is chosen as the field of study for this research. Even keystroke has proven to be effective in authentication of an individual. There is one prominent disadvantage mentioned in [2]. Keystroke patterns vary according to one's mood. If this hypothesis is true, then using keystroke dynamics as a mean of authentication will certainly not prove to be effective and will be invalid. Our study aims to find out whether this hypothesis is true. If yes, then which dimension of emotions are responsible for changing keystroke dynamics.

\section{RELATED WORK}

The work in [1] describes the concept based on using standard input devices, such as keyboard and mouse, as sources of data recognition of user's emotional states. Applications do not adapt to user's context. User context includes information such as their location, emotional states, or situation. This system using key strokes is more intuitive, unobtrusive and has a wider range of users. In the present paper, the emotional 
states are investigated via keystroke dynamics. The proposed method is based on to calculate the pressing time, dwell time, mean time, range and standard deviation time of keystrokes. In this paper, they have proposed keystroke dynamics based application for recognizing emotional states of computer user. This method is inexpensive and non-intrusive to user. For keystroke dynamics method, timing features of fixed texts has been analyzed because fixed text showed better results than free text.

The authors of [2] have found about the research done in static authentication of keystroke dynamics and the future scope as well as the disadvantages of keystroke dynamics. They have listed out the various researches done on keystroke dynamics with the help of statistical and neural network classification techniques. It has been stated that it is impossible to compare both the techniques on the same standards. However, statistical approach is more popularly used pertaining to the fact that it is relatively simple, has less overhead and is easy to implement. On the other hand, neural network gives more accurate results. Through the study, it is found that keystroke dynamics has various advantages making it the best and cheapest authentication technique. But it has a major problem of being inconsistent. Typing pattern of a user can become erratic due to many reasons like being tired or injured or under medication. Emotions play an important role in keystroke dynamics and it changes the typing pattern when a person is flustered or angry. Typing pattern of an individual also changes gradually over time.

The authors of [3] say that currently people store a lot of sensitive data on their mobile devices. Moreover, touch screen allows adding features ranging from pressure of the screen or finger area to the classical time-based features used for keystroke dynamics. In this paper, they examine the effect of these additional touch screen features to the identification and verification performance by using time based features to find out the results. An Android application having its own software keyboard was developed for data collection where users had to introduce some personal data, such as gender, birth date and their experience level regarding smart phone usage in the registration phase. The majority of participants completed 2 sessions in a period of two weeks. The Measurements phase involved user identification measurements that were performed using WEKA (version 3.6.11), a popular machine learning software and used various machine learning methods such as Naive Bayes, Bayesian Network, Nearest neighbor, Decision Trees and Multilayer Perceptron (MLP). This paper demonstrates experimentally that touch screen based features improve the result and enhance the accuracy of both processes.

In [4] a user verification system on mobile phones is proposed. This system is based on behavioral biometric traits which is keystroke dynamics derived from a touchable keyboard. A mobile application is developed for collecting those touch keystroke dynamics. The Median Vector Proximity classifier is applied on the touch keystroke data (touchable keyboard) and the performance of the system is investigated using different number of features. In this paper, they proposed to apply the Median Vector Proximity classifier on the touch keystroke data derived from mobile's touch screen. They evaluated the system on no specific text and extracted 31 and 33 touch features. The average EER were $12.9 \%$ and $12.2 \%$ respectively. They found that the average EER was reduced by about $0.7 \%$. Therefore, the more features they used resulted in more accurate systems. As a future work, they will apply different classifiers on those features and find out which classifier would give better results.

The authors of [5] say that the automatic emotion recognition technology is an important part of building intelligent systems to prevent the computers acting inappropriately. A novel approach for recognizing emotional state by their keystroke typing patterns on a standard keyboard was developed in recent years. However, there was very limited investigation about the phenomenon itself in the previous literatures. Hence, in their study, they conduct a controlled experiment to collect subjects' keystroke data in the different emotional states induced by facial feedback. They examine the difference of the keystroke data between positive and negative emotional states. The results imply that when subjects were in different emotional states, they pressed the keyboard with different strength and demonstrated the tendency of the data on supporting the hypothesis. By conducting the controlled experiment, they validated the hypothesis about the existence of the difference on typing pattern between two opposite emotional states. The keystroke data were also applied in authentication system in previous studies to make the system more secure from hacking.

The work in [6] provides us with the results of a controlled experiment that was performed in which keystroke data of different users were collected in different emotional states induced by International Affective Digitized Sounds (IADS).The authors proposed an experiment designed to examine the effect of film-induced emotional states (PVHA, PVLA, NVHA, NVLA and NVNA $(\mathrm{P}=$ positive, $\mathrm{N}=$ negative, $\mathrm{H}=$ high, $\mathrm{L}=$ low, $\mathrm{n}=$ neutral, $\mathrm{V}$ =valence, $\mathrm{A}=$ arousal) in subjects, with the keystroke dynamics in regard to keystroke rate per second, average duration of keystroke (from key-down until key-up event). The results of their experiment using the fix target typing text and the 63 stimuli selected from the IADS-2 database supports the hypotheses that the keystroke duration and latency are influenced by arousal. Shorter keystroke duration was found when arousal was high compared to the keystroke duration when arousal was low. The result is in line with the findings reported by, which suggest a longer keystroke duration accompanied with negative emotional state. In addition, they found a slowest keystroke latency when arousal is medium. Their findings support the conclusion that the keystroke duration and latency are influenced by arousal and valence. This experiment was conducted in a PC using a keyboard.

The authors of [7] have simplified two main problems with current approaches for identifying emotions which are invasive and costly by using keystroke dynamics. They have conducted a field study where they collected participants' keystrokes and their emotional states via self-reports like questionnaires. Using an experience-sampling approach, users labelled the data with their level of agreement with 15 emotional states and provided additional keystrokes by typing fixed pieces of text. From that data, they extracted keystroke features and created classifiers for 15 emotional states. The results include 2-level classifiers for confidence, hesitance, nervousness, relaxation, sadness, and tiredness with accuracies ranging from 77 to $88 \%$ and show promise for anger and excitement, with accuracies of $84 \%$. Some betterments to this approach are using a validated emotional state scale and distinguishing between different emotions rather than levels of a single emotion. 


\section{OUR SYSTEM}

In our system, the stimuli used had 63 sounds selected from the IADS-2 database, which is developed and distributed by the NIMH Centre for Emotion and Attention (CSEA) at the University of Florida. The IADS-2 database contains various affective sounds proved to be capable of inducing diverse emotions in the affective space. The sounds used as the stimuli were selected from IADS-2 database complying the IADS-2 sound set selection protocol. The protocol includes the constraint about the number of sounds used in a single experiment, and the distribution of the emotions that are expected to be induced by the selected sounds. The physical properties of these sounds were controlled to prevent clipping, and to control for loudness.

\subsection{Data Collection and Pre- Processing}

A subject was made to wear earphones during the experiment and was instructed to type-in a target typing text "748596132" once immediately after hearing each of the International Affective Digitized Sounds 2nd edition (IADS-2) sounds, for 63 trials. The experiment was conducted based on a simple dimensional view of emotion, which assumes that emotion can be defined by a coincidence of values on two different strategic dimensions that are, valence and arousal. To assess these two dimensions of the affective space, the SelfAssessment Manikin (SAM) [8][9], an affective rating system was used to acquire the affective ratings. To collect the data we developed an Android application called Keydroid. Each trial in the Keydroid began with an instruction ("Please type-in the target typing text after listening to the next sound") presented for 5 seconds. Then, the sound stimulus was presented for 6 seconds. After the sound terminated, the SAM with a rating instruction ("Please rate your feeling on both the two dimensions after typing the target typing text '748596132"') was presented. The subject was asked to first type-in the target typing text once, and then make his/her ratings of valence and arousal. A standard $15 \mathrm{~s}$ rating period was used, which allows ample time for the subject to make the SAM ratings. The keystroke data was recorded during the typing task. In addition to the 63 trials, 3 practice trials and a training section was applied prior to the experiment. Three sounds (birds, female sigh, and baby cry) provided the subject with a rough range of the types of the contents that were presented. After these practice trials was the training section, in which the subject continually typed-in the target typing text using the number pad on the application. A number sequence was used as the target typing text instead of an alphabet sequence or symbols to avoid possible interference caused by linguistic context to the subject's emotional states. A comparison of keystroke typing between emotional states using different number sequences may reduce the power of statistical tests (given a same number of trials). Hence, to conduct a more conservative comparison across emotion and to enhance the generalizability of this study, we decided to use a single number sequence that is designed to be general. The target typing text was decided as " 748596132 " to

1) be easy to type without requiring the subjects to perform abrupt changes in their posture

2) have the number of digits fairly distributed on a number pad

3) encourage all the subjects to maintain a same posture (i.e., in terms of finger usage when typing the given sequence.

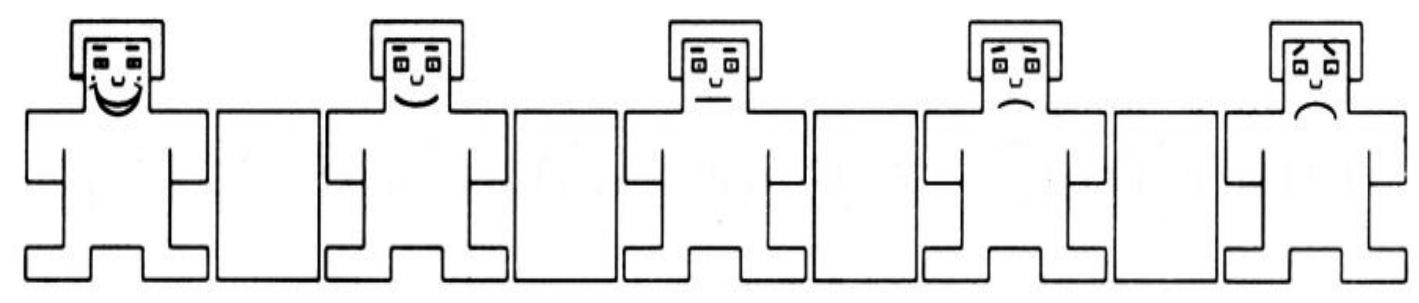

Fig 2: SAM ratings for Valence

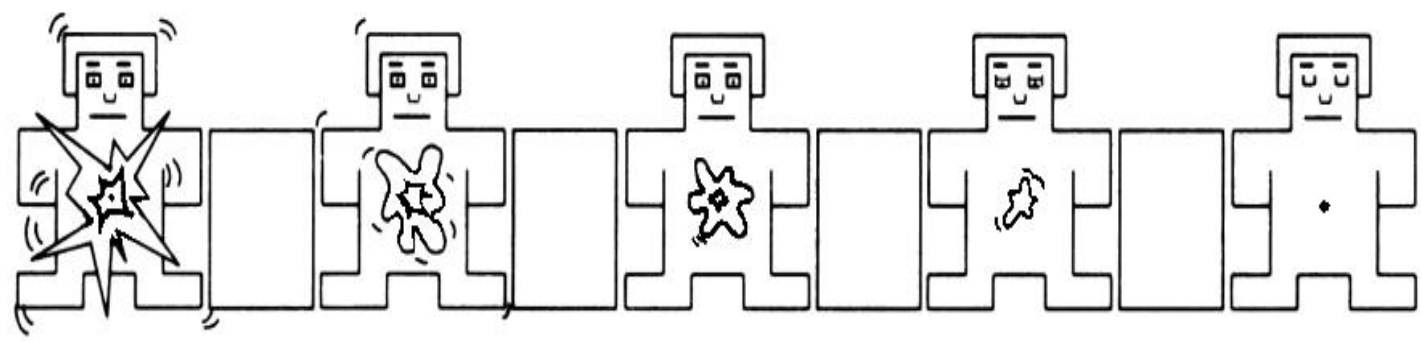

Fig 3: SAM ratings for Arousal

\subsection{Calculations}

The emotions of a subject was predicted based on relation between valence and arousal. IADS-2 sounds elicited that the subjects' feelings of being annoyed or alarmed (i.e. reporting negative valence with medium arousal), but not being angry (i.e. reporting negative valence with high arousal) and not being tired, sad, or bored (i.e. reporting negative valence with low arousal). These values are useful to identify different emotional states. After the data collection, Analysis of variance (ANOVA) was used to calculate the effect of emotions on keystroke patterns. ANOVA is a collection of statistical models used to analyze the differences among group means and their associated procedures (such as "variation" among and between groups). Mainly the change in the keystroke duration based on different arousal and valence ratings was observed. The variance was conducted between arousal, valence, keystroke latency, duration to get the appropriate results. 


\section{RESULTS}

During the comparison of the first and last sample of each of the 23 subjects, it was observed that keystroke latency was decreased by a mean of 2.6 seconds. One of the main reason for this was that the subject became well versed with the typing pattern and typed the sequence faster than the first sample. It was also found that for low or negative valence the mean keystroke latency was the highest of 2.11 seconds. This indicated that the subject takes longer to type when he is bored or in an unpleasant mood.

Through ANOVA we can observe the values of sigma for different dependent variables. If sigma is below 0.05 , it indicates that there is an influence of the source on the dependent variable. From Table 1, it is clear that valence and arousal influence Keystroke Latency. From Table 2, we can infer that Valence and Valence * Arousal together affect Keystroke Duration.

Table 1. ANOVA results of showing influence of Valence and Arousal on Keystroke Latency.

\begin{tabular}{|c|c|}
\hline Dependent Variable: & Keystroke Latency \\
\hline Source & Sigma \\
\hline Valence & 0.037 \\
\hline Arousal & 0.011 \\
\hline Valence*Arousal & 0.299 \\
\hline
\end{tabular}

Table 2. ANOVA results of showing influence of Valence and Valence*Arousal on Keystroke Duration

\begin{tabular}{|c|c|}
\hline Dependent Variable: & Keystroke Duration \\
\hline Source & Sigma \\
\hline Valence & 0.000 \\
\hline Arousal & 0.821 \\
\hline Valence*Arousal & 0.000 \\
\hline
\end{tabular}

\section{FUTURE SCOPE}

The current system of measuring depression is tedious. The present system includes a survey with Likert's Management System. This cannot be an effective way of studying the influence of emotions; a lot of context is lost in the syntax and semantics of the questions asked to the patient or the patient's understanding of the questions. Since auditory stimuli is effective in changing the keystroke of a person, and with keystroke dynamics we can guess the emotions of the person, the amount of depression levels in a patient can be deduced without the complexity of filling endless surveys. However, this will require a few improvements in the current system. Social media gives a lot of importance to user data. The user's emotions can play a huge role in recommendations that should be presented to the user. Currently Facebook asks the user to enter his/her mood i.e. feeling happy, feeling angry etc. Our system will not require to enter his/her emotions on their own. Rather, judge their emotions through their keystroke. Since this is just a future scope. It will require multiple improvements in order to develop this solution further to suit the application.

\section{CONCLUSION}

The current experiment aimed to prove two hypotheses that were; the relationship between keystroke duration and emotion and the relationship between keystroke latency and emotion. The work presented here proved the effect of both valence and arousal in Keystroke Latency and the effect of valence in Keystroke Duration. However, the effect of arousal on Keystroke duration was not proved. One of the main reason was that there were considerably less number of samples collected for high arousal. Future work in this genre should make sure that there are more number of samples collected in high arousal values. Thus this experiment was able to prove the effect of both valence and arousal in Keystroke Latency and the effect of valence in Keystroke Duration.

\section{REFERENCES}

[1] P. V. Shivshankar Rajput, "Implementation of keystroke dynamics application for identifying emotional state," May 2015.

[2] Pranit Shinde, Saideep Shetty, Mahendra Mehra, "Survey of Keystroke Dynamics as a Biometric for Static Authentication," April 2016.

[3] I. L. Margit ANTAL, LászlóZsolt SZABÓ, "Keystroke dynamics on android platform," October 2014

[4] L. A. E. Shatha J. Alghamdi, "Dynamic user verification using touch keystroke based on medians vector proximity," Computational Intelligence, Communication Systems and Networks (CICSyN), June 2015.

[5] T.-C. H. Po-Ming Lee, "The effect of emotion on keystroke: An experimental study using facial feedback hypothesis," Plos One, June 2015.

[6] T.-C. H. Po-Ming Lee, Wei-HsuanTsui, "The influence of emotion on keyboard typing:An experimental study using auditory stimuli," Plos One, June 2015.

[7] Clayton Epp, Michael Lippold, Regan L. Mandryk, "Identifying emotional states using keystroke dynamics"

[8] P. J. Lang, "Behavioral treatment and bio-behavioral assessment: Computer applications," J. B. Sidowski, J. H. Johnson, T A. Williams (Eds.), Technology in mental health care delivery systems (pp. 119-137), 1980.

[9] L. P. J. Bradley, M. M., "Measuring emotion: The selfassessment manikin and the semantic differential," Journal of Behavioral Therapy and Experimental Psychiatry, 25(1), 49-59., 1994. 